\title{
Performance Analysis of Fractional-Order PID Controller for a Parabolic Distributed Solar Collector
}

\author{
Shahrazed Elmetennani, Ibrahima N'Doye, Khaled Nabil Salama and Taous-Meriem Laleg-Kirati \\ \{shahrazed.elmetennani, ibrahima.ndoye, khaled.salama, taousmeriem.laleg@kaust.edu.sa\}
}

\begin{abstract}
This paper studies the performance of a fractional-order proportional integral derivative (FOPID) controller designed for parabolic distributed solar collectors. The control problem addressed in concentrated solar collectors aims at forcing the produced heat to follow a desired reference despite the unevenly varying solar irradiance. In addition to the unpredictable variations of the energy source, the parabolic solar collectors are subject to inhomogeneous distributed efficiency parameters affecting the heat production. The FOPID controller is well known for its simplicity with better tuning flexibility along with robustness with respect to disturbances. Thus, we propose a control strategy based on FOPID to achieve the control objectives. First, the FOPID controller is designed based on a linear approximate model describing the system dynamics under nominal working conditions. Then, the FOPID gains and differentiation orders are optimally tuned in order to fulfill the robustness design specifications by solving a nonlinear optimization problem. Numerical simulations are carried out to evaluate the performance of the proposed FOPID controller. A comparison to the robust integer order PID is also provided. Robustness tests are performed for the nominal model to show the effectiveness of the FOPID. Furthermore, the proposed FOPID is numerically tested to control the distributed solar collector under real working conditions.
\end{abstract}

Index Terms-Parabolic distributed solar collector, fractional-order PID (FOPID), robust PID, process control, robustness analysis, PID, iterative optimization.

\section{INTRODUCTION}

Solar energy is a promising available clean source to respond to the exponential growth of energy demand while reducing the dependence on fossil resources and their environmental impact. In particular, the parabolic distributed technology has been widely used in solar thermal plants. A concentrated distributed solar collectors consists of parabolic shaped mirrors which concentrate the received sunlight into a receiver tube placed in the central line of the collector in order to heat the thermal carrier fluid flowing in it [1], [2]. The control challenge in these systems consists in managing the heat production despite the external disturbances, mainly the solar irradiance. The system is controlled by tuning the fluid flow rate to force the temperature at the collector outlet to track a desired reference. The parabolic troughs are spatially distributed processes, described by a nonlinear model of infinite dimension (first order hyperbolic Partial Differential Equation PDE) with limited measurements [1],

Shahrazed Elmetennani, Ibrahima N'Doye, Khaled Nabil Salama and Taous-Meriem Laleg-Kirati are with Computer, Electrical and Mathematical Sciences and Engineering Division (CEMSE), King Abdullah University of Science and Technology (KAUST).
[3]. Standard control strategies fail to provide good performance particularly under changing working conditions due to scattered clouds and the uncleanness of the mirrors. Hence, extrapolating only available measurements might not be a reasonable assumption. Therefore, a good control strategy for distributed solar collectors should be able to deal with the issue related to the solar irradiation intermittency and variations of the mirrors efficiency. For this purpose, numerous control techniques have been combined with a feedforward controller, estimation or identification methodologies such as the indirect adaptive control [4], [5], model predictive controllers [6], [7], [8], adaptive fuzzy switching control techniques [9] or more advanced adaptive techniques [10], [11]. The adaptive based approach is usually affected by modeling uncertainties which affects the performance of the controller.

In this study, the FOPID controller is introduced as an alternative robust control strategy to the PID control [12], [13], [4]. The classical PID controller is one of the most used controller in industry for closed control-loops thanks to its simplicity in real time implementation. The design and tuning of such a controller has been widely covered and still represents an active field of research especially for industrial plants subject to external disturbances, see [14], [15]. Indeed, due to the significant variations in the heat transfer dynamics affected by the external disturbances, satisfactory performance covering the total range of disturbances is difficult to reach with a classical PID without an external compensation. Thereafter, it is desirable to design robust control strategies without additional computational effort.

The FOPID controller is a generalization of the PID controller by considering fractional derivatives and integral actions [16]. In recent years, fractional calculus has gained significant attention in various applications in engineering and science. This interest is driven by the possibility to obtain accurate mathematical models of real objects, especially for those presenting memory, hereditary, or self-similarity properties [16]. Besides of modeling, the fractional calculus has been applied for control design. The FOPID offers better tuning flexibility thanks to the additional differentiation orders allowing for better control performance and robustness. Several studies have addressed the properties of the FOPID and compared its performance to the classical PID under different contexts [17], [18], [19], [20], [21], [22], [23]. Different tuning procedures for the FOPID have been proposed in the literature where the main objective is 
to optimally tune the gains and the differentiation orders. Examples of these tuning procedures include: the procedure presented in [17], which is based on solving a set of nonlinear equations. In [18] and [19] a tuning rule for a fractionalorder controller with application to motion systems is given. Genetic algorithms are used in [20] to design a FOPID controller. Another class of fractional controllers is proposed by [21]. This class of controllers is more appropriate to ensure robustness of the closed-loop to static gain variations with a conventional CRONE template [22]. Some efficient design techniques have also been proposed and are summarized in [17], [23].

In this paper, given a nominal approximate model of the solar collector, we design a FOPID control to manage the heat production. The gains and differentiation orders are tuned by solving a nonlinear optimization problem. A numerical optimization algorithm implemented in Matlab environment is proposed. The requirements to be satisfied by the controller are expressed in order to fulfill some frequency-domain design specifications. The proposed FOPID is implemented to test both, the nominal model and the solar collector model, in closed loop. The performance of the FOPID is evaluated by comparison to the robust PID. Moreover, robustness analysis is performed with respect to static gain variations, load disturbance effect, high frequency noise rejection and output disturbance rejection. To the best of our knowledge, the optimization procedure which combine two types of optimization algorithms and the use of FOPID controller in solar collector application have not been proposed.

This paper is organized as follows. In section II, some preliminary results about fractional control are presented. In section III, the solar plant is described and the control problem is introduced. In section IV, the design and the tuning procedure of the FOPID controller are given. Results of the numerical tests are illustrated and discussed in section $\mathrm{V}$ followed by concluding remarks in section VI.

\section{Preliminaries}

Fractional calculus is a generalization of differential and integral operators to a non-integer order fundamental operator ${ }_{0} D_{t}^{\alpha}$ where $\alpha$ is the operator order and $t$ denotes the limit of the operation. Several definitions of this fractional integro-differential operator exist. However, we restrict our attention to one particular definition, which is used throughout this work for evaluating fractional-order systems. It is the Grünwald-Letnikov definition [16], [23].

Definition 1: The Grünwald-Letnikov definition is given as follows:

$$
{ }_{0} D_{t}^{\alpha} f(t)=\frac{d^{\alpha} f(t)}{d t^{\alpha}}=\lim _{t \rightarrow 0} h^{-\alpha} \sum_{j=0}^{\left[\frac{t}{n}\right]}(-1)^{j}\left(\begin{array}{c}
\alpha \\
j
\end{array}\right) f(t-j h),
$$

with $n \in \mathbb{N}, \alpha \in \mathbb{R}^{+}$, [.] denotes the integer part, $t=k h$ is the number of steps, and $h$ the step size.

Assuming zero initial conditions, the Laplace transform of the fractional derivative with $\alpha \in \mathbb{R}^{+}$, is given by:

$$
\int_{0}^{\infty} e^{-s t}{ }_{0} D_{t}^{\alpha} f(t)=s^{\alpha} F(s)
$$

where $s=j \omega$ is the Laplace transform variable. Thus a fractional-order differential equation can be expressed in transfer function form in the frequency domain as follows:

$$
P(s)=\frac{b_{m} s^{\beta_{m}}+b_{m-1} s^{\beta_{m-1}}+\cdots+b_{0} s^{\beta_{0}}}{a_{n} s^{\alpha_{n}}+a_{n-1} s^{\alpha_{n-1}}+\cdots+a_{0} s^{\alpha_{0}}} .
$$

\section{CONTROL PROBLEM FORMULATION}

A parabolic distributed solar collector is an industrial system producing thermal energy using heat transport in a thermal carrier fluid. The collector concentrates the received sunlight with its parabolic shaped mirrors to the central tube where the fluid flows (see Fig.1).

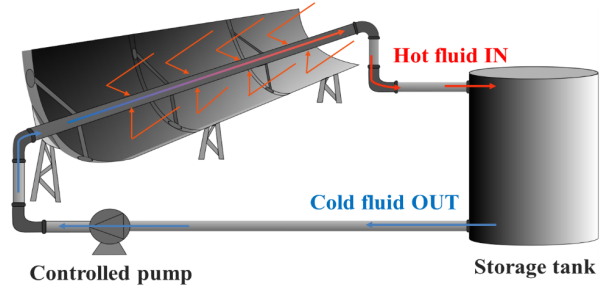

Fig. 1. Distributed solar collector

Linear black-box models have been proposed in the literature to approximate the collector dynamics ([1]). For control purposes, low order linear models have been commonly used [4], [6], [24]. In this study, the FOPID is designed based on an approximate transfer function satisfying some performance criteria in the frequential domain. Therefore, the heat transfer dynamics are described by a first order transfer function. The parameters of the considered linear approximate model are identified using data from a numerical test under real working conditions. A set of input/output data has been generated using the Eulerian approximation of the distributed model with 500 states. The numerical test for data generation has been conducted using the first order hyperbolic partial differential equation describing the conservation of the energy where the system's state is the fluid temperature (3) [1], [3]:

$$
\left\{\begin{array}{l}
T_{t}(x, t)+u(t) T_{x}(x, t)=\underline{I}(t) \\
T(0, t)=T_{\text {in }}(t) \\
y(t)=T(L, t)=T_{\text {out }}(t)
\end{array}\right.
$$

where $x \in[0, L]$ denotes the position along the pipe and $t \in$ $\mathbb{R}^{+}$represents the time. $T(x, t)$ refers to the fluid temperature at a certain position $x$ and time $t$, where $T_{t}(x, t) \equiv \frac{\partial T(x, t)}{\partial t}$ and $T_{x}(x, t) \equiv \frac{\partial T(x, t)}{\partial x}$ are the first derivatives with respect to time and space respectively.

$\underline{I}(t)=\frac{\mathbb{N} u_{0} G}{\mathbb{R} h o c A_{s}} I(t)$ is the source term depending on the solar irradiance $I(t) \cdot u(t)=\frac{Q(t)}{A_{s}}$ is the system control input which is function of the fluid volumetric flow rate $Q(t)$. Besides, $T_{\text {in }}$ and $T_{\text {out }}$ denote the measured boundary values of the fluid temperature respectively at the positions $x=0$ and $L$ such that $y(t)=T_{\text {out }}$ is the variable of interest to be controlled. The remaining system parameters are summarized in Table I.

For control purposes, the distributed solar plant can be approximated by a linear system when considering small disturbances [1]. Observations of step responses between the system output $y(t)$ and an auxiliary input $\bar{u}$ of the solar 
TABLE I

PARAMETERS OF THE SOLAR COLLECTORS FIELD.

\begin{tabular}{lll}
\hline Symbol & Measure unit & Description \\
\hline$t$ & $s$ & Time \\
$x$ & $m$ & Space \\
$T(x, t)$ & ${ }^{\circ} C$ & Fluid temperature \\
$I(t)$ & $W / m^{2}$ & Solar irradiance \\
$Q(t)$ & $\mathrm{m}^{3} / \mathrm{s}$ & Fluid flow rate \\
$\mathbb{R} h o$ & $\mathrm{~kg} \mathrm{~m}^{-3}$ & Fluid density \\
$c$ & $J C^{-1} \mathrm{~kg}^{-1}$ & Fluid specific heat capacity \\
$A_{s}$ & $\mathrm{~m}^{2}$ & Tube cross-sectional area \\
$\mathbb{N} u_{0}$ & & Mirrors optical efficiency \\
$G$ & $m$ & Mirrors optical aperture \\
$L$ & $m$ & Length of the collector tube
\end{tabular}

plant show that its behavior can be identified using Matlab environment by a first-order transfer function.

$$
P(s)=\frac{y(p)}{\bar{u}(p)}=\frac{K}{1+T s},
$$

where $s$ is Laplace operator and

$$
\bar{u}(t)=\frac{1}{u(t)},
$$

such that the control input $u$ is constrained by the physical limitations of the system:

$$
0<u_{\min }<u(t)<u_{\max } .
$$

The identification leads to the following transfer function which is used as a ground for the control design in this paper.

$$
P(s)=\frac{160.2}{1+6.667 s}
$$

\section{FOPID CONTROL DESIGN}

The transfer function corresponding to the parallel form of the FOPID where the integral component is of order $\lambda$ and the derivative component is of order $\mu$ has the following form in the Laplace domain:

$$
C(s)=K_{p}+\frac{K_{i}}{s^{\lambda}}+K_{d} s^{\mu}, \quad(\lambda, \mu>0),
$$

where $K_{p}$ is the proportional gain, $K_{i}$ and $K_{d}$ are the integration and the differentiation gains consecutively.

Several methods for the FOPID controller tuning have been proposed (see [17], [23], [25], [26], [27] and references therein). Numerical optimization based methods are regularly used to solve this problem [27].

In this study, our objective is to design a FOPID control based on numerical optimization in order to study its performance in the control of distributed collectors under varying working conditions. The tuning procedure is based on satisfying a set of equations derived to achieve the control objectives under the frequency-domain robustness constraints. Frequency domain based design of the FOPID controller was first proposed by Monje et al. [17] resorting to a constrained optimization problem.

We consider the fractional orders for the integral and the derivative actions $\lambda$ and $\mu$ of the FOPID controller to be such that $0<(\lambda, \mu)<1$. If $P(s)$ is the model of the process plant, then the objective is to find a controller $C(s)$, such that the open loop system $G(s)=C(s) P(s)$ would meet the following design specifications:

- Gain cross-over frequency specification

$$
\left|G\left(j \omega_{g c}\right)\right|_{d B}=\left|C\left(j \omega_{g c}\right) P\left(j \omega_{g c}\right)\right|_{d B}=0 \mathrm{~d} B .
$$

- Phase margin specification

$$
\arg \left[G\left(j \omega_{g c}\right)\right]=\arg \left[C\left(j \omega_{g c}\right) P\left(j \omega_{g c}\right)\right]=-\pi+\phi_{m},
$$

where $\phi_{m}$ is related to the damping of the system.

- Robustness to gain variation

$$
\left(\frac{d}{d \omega}\left(\arg \left[G\left(j \omega_{g c}\right)\right]\right)\right)_{\omega=\omega_{g c}}=0 .
$$

This condition forces the phase to be flat at $\omega_{g c}$ or almost constant within an interval around $\omega_{g c}$.

- Complementary sensitivity specification

$$
\begin{gathered}
|T(j \omega)|=\left|\frac{C(j \omega) P(j \omega)}{1+C(j \omega) P(j \omega)}\right|_{\mathrm{d} B} \leqslant A \mathrm{~d} B \quad \forall \omega \geqslant \omega_{t} \\
\Longrightarrow\left|T\left(j \omega_{t}\right)\right|=A \mathrm{~d} B
\end{gathered}
$$

where $A$ is the magnitude or noise attenuation for frequencies $\omega \geqslant \omega_{t} \mathrm{rad} / \mathrm{s}$.

- Sensitivity specification

$$
\begin{gathered}
|S(j \omega)|=\left|\frac{1}{1+C(j \omega) P(j \omega)}\right|_{\mathrm{d} B} \leqslant B \mathrm{~d} B \quad \forall \omega \leqslant \omega_{s} \\
\Longrightarrow\left|S\left(j \omega_{s}\right)\right|=B \mathrm{~d} B
\end{gathered}
$$

where $B$ is the magnitude or load disturbance suppression for frequencies $\omega \leqslant \omega_{s} \mathrm{rad} / \mathrm{s}$.

- Elimination of steady-state error: the steady-state error of the closed loop system automatically gets canceled with the introduction of the fractional integrator.

The five parameters of the FOPID controller (8) can now be tuned using the five specifications (9)-(13). Here, the numerical optimization algorithm, namely of Nelder-Mead's simplex method, has been implemented using the Matlab function fmincon. In practice most solutions found with this optimization method are good enough, but they strongly depend on initial estimates of the provided parameters. To ensure that the global minimum is reached by the optimization process, we combined two types of optimization algorithms. We performed first the genetic algorithm and used the obtained results as the initial search vector for fmincon algorithm. To the best of our knowledge, the resulting two optimization algorithms procedure has not been proposed for fractional-order PID controller.

\section{NUMERICAL SIMULATIONS}

This section evaluates the designed FOPID performance for the closed loop control of the nominal approximate model (7) considering different types of disturbances. Then, the FOPID is implemented for the solar collector plant in closed loop under real working conditions.

After characterization of the solar collector plant, the resulting transfer function takes the form of a first order transfer function (7). 
The system's desired performance defining the constrained optimization problem are described by the following specifications:

- gain crossover-frequency, $\omega_{g c}=1 \mathrm{rad} / \mathrm{s}$;

- phase margin, $\phi_{m}=90^{\circ}$;

- robustness to variations in the gain of the plant must be fulfilled;

- noise rejection: $|T(j \omega)| \leqslant-10 \mathrm{~d} B, \forall \omega \geqslant \omega_{t}=10 \mathrm{rad} / \mathrm{s}$;

- sensitivity function: $|S(j \omega)| \leqslant-20 \mathrm{~d} B, \forall \omega \leqslant \omega_{s}=$ $0.01 \mathrm{rad} / \mathrm{s}$;

For the numerical tests, we propose two different sets of parameters depending on the tuning rules to test the closed loop performance. Two parameters sets have been generated using the constrained optimization algorithm. The first set is the result of the optimization of the five parameters (gains and fractional orders) leading to a FOPID controller. The second set has been derived by optimizing the gains only while considering integer order for the integration and the derivation leading to a robust PID (ROPID) controller.

The optimization procedure for both sets has been run by setting the following ranges for the gains and orders:

$$
\begin{gathered}
K_{p}=[0.01 ; 10] \quad K_{i}=[0.001 ; 10], \quad K_{d}=[0.01 ; 10], \\
\lambda=[0.1 ; 1] \quad \text { and } \mu=[0.1 ; 1] .
\end{gathered}
$$

The following FOPID controller parameters obtained from the two cases:

- Optimization of all parameters (FOPID):

$$
\begin{aligned}
& K_{p}=0.03370, \quad K_{i}=0.0082, \quad K_{d}=0.0101, \\
& \lambda=0.9357, \quad \mu=0.10 .
\end{aligned}
$$

- Optimization of the gains only (robust PID):

$$
K_{p}=0.02, K_{i}=0.01, K_{d}=0.01, \lambda=\mu=1 .
$$

A. Performance of the controller subject to nominal conditions

The resulting two controllers are tested in closed loop to control the first order system (7) to simulate the plant under nominal conditions. The obtained dynamic responses are presented and compared in Fig. 2. Further comparative details are explored in Table II. Observing these results, we can conclude that the FOPID controller presents better performance compared to the robust PID in terms of rising time, settling time, overshoot and steady state error (Ess). The comparative performance evaluation has been also studied in the frequency domain. The Bode diagrams of the openloop system $G(s)=C(s) P(s)$ are shown in Fig. 3. As it can be seen, the gain crossover frequency specification $\omega_{g c}=1 \mathrm{rad} / \mathrm{s}$ and the phase margin specification $\phi_{m}=90^{\circ}$ are fulfilled for the FOPID controller. TABLE II

PERFORMANCE ANALYSIS

\begin{tabular}{l||lll} 
Controllers & Rise time Settling time Ess Overshoot
\end{tabular}

\begin{tabular}{|l|cccc|}
\hline FOPID (all parameters) & 2.0332 & 3.4481 & $3.96 e^{-4}$ & $0 \%$ \\
\hline Robust PID (gains only) & 2.9209 & 11.4935 & $5.79 e^{-4}$ & $12.58 \%$ \\
\hline
\end{tabular}

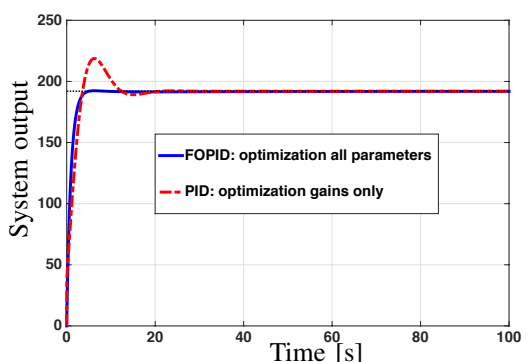

Fig. 2. Reference tracking : step responses comparison.

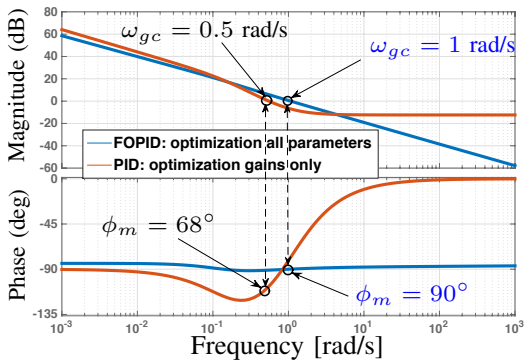

Fig. 3. Bode diagrams of the open-loop system $G(s)=C(s) P(s)$.

\section{B. Robustness with respect to gain and load variations}

In the second test in this study, our objective is to test the robustness of the FOPID with respect to static gain and load variations. Figs. 4 - 5 show the closed loop response of the system subject to gain variations and a load input excitation using the two controllers. We observe that the tracking responses and disturbance rejection of the FOPID controller remain almost constant under $\pm 18.6 \%$ gain variations compared to the robust PID controller. Comparative details are explored in Table III. As it can be observed the maximum percent overshoot deviation of the FOPID controller do not change with $\pm 18.6 \%$ gain variations from the optimal point whereas the ROPID controller varies almost $\pm 5 \%$ from its optimal point, which means that the controlled system using FOPID is more robust to gain changes. TABLE III

Performance analysis to gain Variations

\begin{tabular}{|c||c||lccc|}
\hline Gain variations & Controllers & Rise & Settling & Ess & Overshoot \\
\hline $\mathrm{K}=160.2$ & FOPID & 2.033 & 3.448 & $3.96 e^{-4}$ & $0 \%$ \\
& Robust PID & 2.920 & 11.493 & $5.79 e^{-4}$ & $12.58 \%$ \\
\hline $\mathrm{K}=130$ & FOPID & 1.759 & 3.025 & $1.162 e^{-4}$ & $0 \%$ \\
& Robust PID & 3.312 & 12.640 & $8.01 e^{-4}$ & $13.23 \%$ \\
\hline $\mathrm{K}=190$ & FOPID & 2.337 & 3.887 & $6.37 e^{-5}$ & $0.006 \%$ \\
& Robust PID & 2.638 & 10.657 & $8.75 e^{-5}$ & $11.95 \%$ \\
\hline
\end{tabular}

C. Robustness to high frequency noise and output disturbance

To assess the robustness of the system response with respect to high frequency noise and output disturbance, we have explored the magnitudes of the functions $T(j \omega)$ and $S(j \omega)$ for the nominal plant given in (7) by analysing their respective Bode diagrams as shown in Figs. 6 and 7 respectively. As it can be observed, $|T(j \omega)|_{d B} \leqslant-10 \mathrm{~d} B, \forall \omega \geqslant$ 


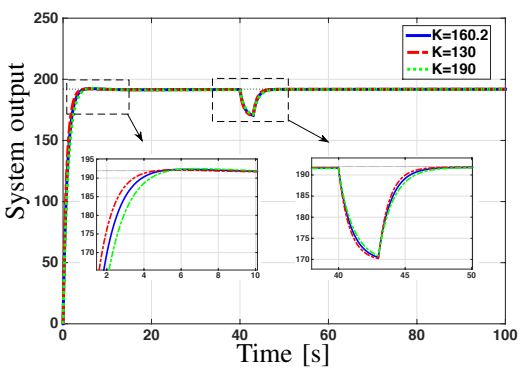

Fig. 4. Step responses of the controlled system with gains variations and load disturbance effect (FOPID controller with optimization all parameters).

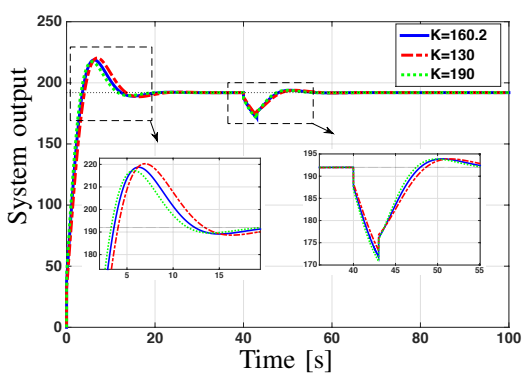

Fig. 5. Step responses of the controlled system with gains variations and load disturbance effect (Robust PID controller with optimization gains only).

$\omega_{t}=10 \mathrm{rad} / \mathrm{s}$, and $|S(j \omega)|_{d B} \leqslant-20 \mathrm{~d} B \forall \omega \leqslant \omega_{s}=$ $0.01 \mathrm{rad} / \mathrm{s}$. We can conclude that both controllers fulfill the specifications but the FOPID controller tends to have more robustness than the ROPID controller as we go far from $\omega_{t}$ and $\omega_{s}$. It means that the FOPID controller has much better robustness than the integer-order ROPID controller presenting a very satisfactory output disturbance rejection and measurement noise rejection.

From the overall results presented above, we observe clearly that FOPID controller is more effective and robust than the ROPID controller for trajectory tracking control in spite of varying disturbances imposing hard constraints on the closed loop performance.

\section{Effect of the fractional orders on the closed loop perfor- mance}

To study the impact of introducing the fractional order PID on the closed loop performance, we have evaluated the frequency response function when one fractional order parameter at a time changes in the FOPID controller. Results are shown in Figs. 8 and 9. The variations of the fractional integrator and derivative orders $\lambda$ and $\mu$ around $\omega_{g c}=1 \mathrm{rad} / \mathrm{s}$ generate changes in the frequency response function (see Figs. 8 and 9). The increment of the fractional integrator and derivative $\lambda$ and $\mu$ lead to a loop gain and phase with almost constant gain crossover frequency $\omega_{g c}=1$ and multiple phase margins (see Figs. 8 and 9). The obtained results show that $\lambda$ affects remarkably the closed loop performance in low frequencies where $\mu$ has an impact on the system response in high frequencies. Thereafter, the fractional orders of the derivative and the integral actions of the FOPID controller give more flexibility for tuning to achieve the desired specifications.

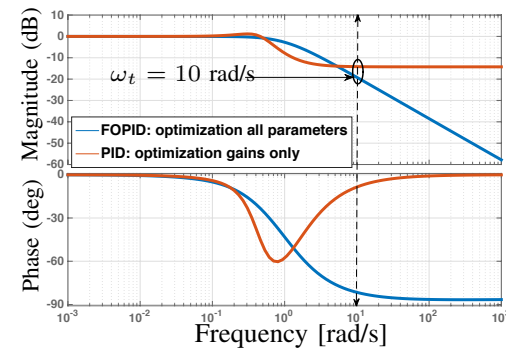

Fig. 6. Bode diagrams of $T(j \omega)$ (noise rejection).

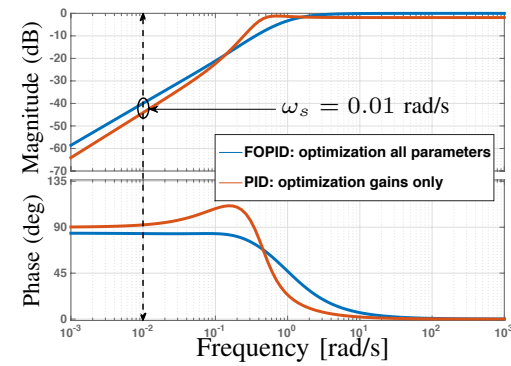

Fig. 7. Bode diagrams of $S(j \omega)$ (sensitivity function).

\section{E. Performance of the controller under real working condi-} tions

After performance evaluation of the designed FOPID for the nominal model, the closed loop system has been tested for the real model of the solar collector presented in (3). The numerical tests have been carried out considering real working conditions for the solar irradiance and the inlet temperature along two hours (see Fig. 10).

The obtained results for the different controllers are shown in Fig. 11. It is clear that the designed FOPID outperforms the classical robust PID. Indeed, the designed FOPID presents interesting features despite the highly varying external disturbances with a relatively short transient time with less oscillations around the reference along with static error converging to zero. We can conclude that the FOPID could achieve the control objectives with respect to static gain variations, load disturbance effect, high frequency noise rejection and output disturbance rejection without added computational complexity for the real time implementation.

\section{CONCLUSION}

In this paper, a FOPID has been proposed to control the concentrated distributed solar collector. The performance of the presented FOPID has been evaluated in closed loop under various working conditions and it has been compared to the integer-order ROPID controller. The simulation results have shown that the FOPID controller is more effective and robust than the integer-order ROPID controller, while it preserves its simplicity of implementation. Moreover, numerical simulations with real process parameters values presented satisfactory results highlighting the effectiveness of the proposed controllers in terms of robustness, tracking precision and time response. 


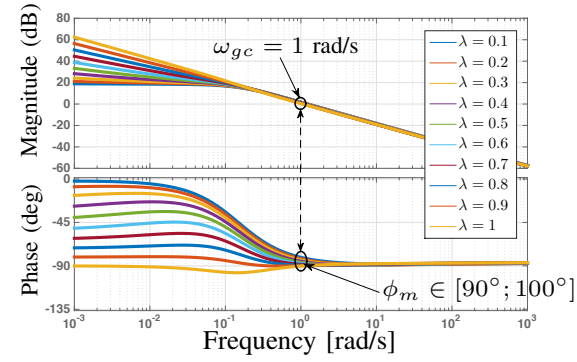

Fig. 8. Bode diagrams of the open-loop system $G(s)=C(s) P(s)$ when the fractional order integral $\lambda$ varies from the robust FOPID controller.

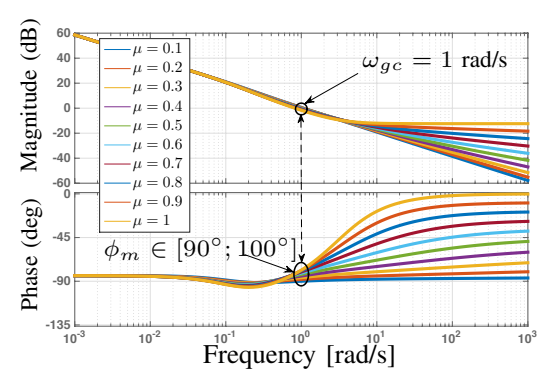

Fig. 9. Bode diagrams of the open-loop system $G(s)=C(s) P(s)$ when the fractional order derivative $\mu$ varies from the optimal FOPID controller.

\section{ACKNOWLEDGMENT}

Research reported in this publication has been supported by the King Abdullah University of Science and Technology (KAUST).

\section{REFERENCES}

[1] E. F. Camacho, M. Berenguel, and F. R. Rubio, Advanced control of solar plants. Springer, 1997.

[2] J. M. Lemos, R. N. Silva, and J. M. Igreja, Adaptive Control of Solar Energy Collector Systems. Springer, 2014.

[3] E. F. Camacho, F. R. Rubio, M. Berenguel, and L. Valenzuela, "A survey on control schemes for distributed solar collector fields. part i: Modeling and basic control approaches," Solar Energy, vol. 81, pp. 1240-1251, 2007.

[4] E. F. Camacho, F. R. Rubio, and F. Hughes, "Self-tuning control of a solar power plant with a distributed collector field," IEEE Control Systems Magazine, vol. 12, pp. 227-238, 1992.

[5] J. M. Igreja, J. M. Lemos, and R. N. Silva, Adaptive receding horizon control of a distributed collector solar field. 44th IEEE Conference on Decision and Control, and the European Control Conference, Seville, Spain, Dec 2005

[6] E. F. Camacho and M. Berenguel, "Robust adaptive model predictive control of solar power plant with bounded uncertainties," Int. Journal of Adaptive Control and Signal Processing, vol. 11, pp. 311-325, 1997.

[7] M. Galvez-Carrillo, R. De Keyser, and C. Ionescu, "Nonlinear predictive control with dead-time compensator: Application to a solar power plant," Solar Energy, vol. 83, pp. 743-752, 2009.

[8] A. J. Gallego and E. F. Camacho, "Adaptive state space model predictive control of a parabolic trough field," Control Engineering Practice, vol. 20, pp. 904-911, 2012.

[9] J. Henriques, A. Cardoso, and A. Dourado, "Supervision and c means clustering of pid controllers for a solar power plant," Int. Journal of Approximate Reasoning, vol. 11, pp. 311-325, 1999.

[10] G. Pin, M. Falchetta, and G. Fenu, "Adaptive time-warped control control of molten salt distributed collector solar fields," Control Engineering Practice, vol. 16, pp. 813-823, 2008.

[11] R. N. Silva, J. M. Lemos, and L. M. Rato, "Variable sampling adaptive control of a distributed collector solar field," IEEE Transactions on Control Systems Technology, vol. 11, pp. 813-823, Sep 2003.

[12] A. J. Gallego and E. F. Camacho, "Estimation of effective solar irradiation using an unscented kalman filter in a parabolic-trough field," Solar Energy, vol. 86, pp. 3512-3518, 2012.

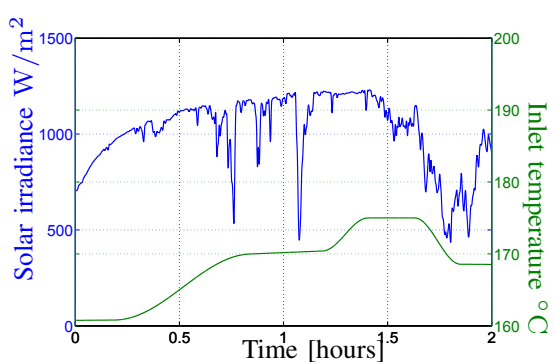

Fig. 10. Profiles of the external disturbances

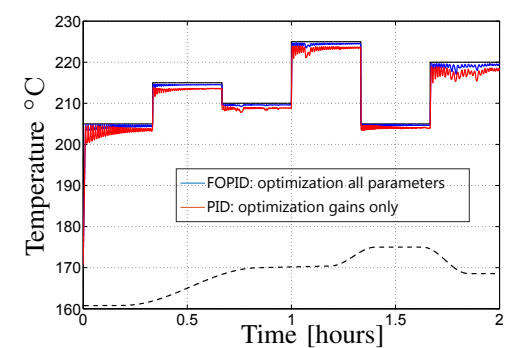

Fig. 11. Reference tracking results for the real system

[13] C. M. Cirre, M. Berenguel, L. Valenzuela, and E. F. Camacho, "Feedback linearization control for a distributed solar collector field," Control Engineering Practice, vol. 15, pp. 1533-1544, 2007.

[14] K. Astrom and T. Hagglund, "The future of PID control," Control Engineering Practice, vol. 9, no. 11, pp. 1163-1175, 2001.

[15] K. Astrom, T. Hagglund, C. C. Hang, and W. K. Ho, "Automatic tuning and adaptation for pid controllers-a survey," Control Engineering Practice, vol. 9, no. 4, pp. 699-714, 1993.

[16] I. Podlubny, Fractional Differential Equations. New York: Academic Press, 1999.

[17] C. Monje, B. Vinagre, V. Feliu, and Y. Chen, "Tuning and autotuning of fractional order controllers for industry applications," Control Engineering Practice, vol. 16, no. 7, pp. 798-812, 2008.

[18] H. S. Li, Y. Luo, and Y. Q. Chen, "A fractional order proportional and derivative (FOPD) motion controller: Tuning rule and experiments," IEEE Trans. Contr. Syst. Techn., vol. 18, pp. 516-520, 2010.

[19] J. Lu and G. Chen, "Robust stability and stabilization of fractionalorder interval systems: an LMI approach," IEEE Trans. Aut. Contr., vol. 54, pp. 1294-1299, 2009.

[20] H. Li, Y. Luo, and Y. Chen, "An output feedback approach to the design of robust fractional PI and PID controllers," International Journal of soft computing and Engineering, no. 3, 2011.

[21] M. Tenoutit, N. Maamri, and J. Trigeassou, "An output feedback approach to the design of robust fractional PI and PID controllers," in World Congress IFAC, (Milan, Italy), 2011.

[22] A. Oustaloup, La Commande CRONE. Paris: Hermes, 1991

[23] C. A. Monje, Y. Q. Chen, B. M. Vinagre, D. Xue, and V. Feliu, Fractional-order systems and controls: Fundamentals and Applications. Berlin: Springer, 2010.

[24] J. Normey-Rico, C. Bordons, M. Berenguel, and E. F. Camacho, "A robust adaptive dead-time compensator with application to a solar collector field," 1998.

[25] F. Padula and A. Visioli, "Tuning rules for optimal PID and fractionalorder PID controllers," Journal of Process Control, vol. 21, pp. 69-81, 2011.

[26] C. Yeroglu, C. Onat, and N. Tan, "A new tuning method for PID controller," in in Proc. Int. Conf. Electrical and Electronics Engineering ELECO, vol. II, pp. 312-316, 2009.

[27] A. Tepljakov, E. Petlenkov, and J. Belikov, "A flexible MATLAB tool for optimal fractional-order PID controller design subject to specifications," in Proc. European Contr. Conf., (Heifi, China), pp. 4698-4703, 2012. 